\title{
Glutathione reductase activity, riboflavin status, and disease activity in rheumatoid arthritis
}

\author{
Diarmuid M Mulherin, David I Thurnham, R Deva Situnayake
}

\begin{abstract}
Objective-To measure erythrocyte glutathione reductase (EGR) activity and riboflavin status, and their relations to disease activity, in rheumatoid arthritis patients compared to healthy controls.

Methods-Patients with rheumatoid arthritis were classified as active if there were features of articular inflammation which required initiation or change of disease modifying therapy, and as inactive if they had little evidence of articular inflammation. EGR was measured in patients and healthy controls by a functional assay with or without the addition of flavin adenine dinucleotide (FAD). The ratio of stimulated EGR (with FAD added) to basal EGR (no added FAD), which measures riboflavin status, is known as the EGR activation coefficient (EGRAC). An EGRAC $\geqslant 1.3$ represents biochemical riboflavin deficiency.
\end{abstract}

Results-91 patients with rheumatoid arthritis, including 57 with active disease, and 220 healthy controls were studied. Both basal and stimulated EGR were significantly higher in patients with rheumatoid arthritis $(P=0.0001)$ than in controls. Biochemical riboflavin deficiency was identified in $41 \%$ controls and $33 \%$ patients with active rheumatoid arthritis but was significantly less frequent $(9 \%)$ in patients with inactive compared to active disease $(P=0.02)$ or healthy controls $(P=$ 0.0006). Pain score, articular index, $C$ reactive protein, and erythrocyte sedimentation rate were increased in patients with riboflavin deficiency (all $P<0.02$ ). Conclusions-Higher basal and stimulated EGR might be expected in patients with rheumatoid arthritis in response to chronic oxidative stress due to synovial inflammation. The association of riboflavin deficiency with increased disease activity suggests that impaired EGR activity could facilitate continuing inflammation in these patients. Key terms: riboflavin deficiency; rheumatoid arthritis; glutathione reductase

\section{(Ann Rheum Dis 1996;55:837-840)}

Reactive oxygen species and other free radicals may contribute to cellular injury in rheumatoid arthritis and other inflammatory diseases." Glutathione, in reduced form (GSH), maintains cell membrane integrity against oxidative stress by acting as a substrate for the reduction of peroxides to less damaging alcohols by glutathione peroxidase. ${ }^{2}$ Glutath- ione reductase facilitates this process by catalysing the conversion of oxidised glutathione (GSSG) to GSH, thereby maintaining an adequate level of intracellular GSH. Optimal glutathione reductase activity depends on adequate availability of flavin adenine dinucleotide (FAD), a coenzyme derived from riboflavin (vitamin B-2). Thus glutathione reductase activity is suboptimal in individuals with riboflavin deficiency. ${ }^{3}$ Erythrocyte GSH and glutathione reductase levels rise in healthy individuals exposed to chronic oxidative stress. ${ }^{4}$ However, in patients with active rheumatoid arthritis, erythrocyte GSH concentrations were not increased but rose when disease activity improved. ${ }^{5}$ These findings suggest that GSH levels may be inappropriately depressed in patients with active rheumatoid arthritis, perhaps reflecting impaired glutathione reductase activity. Under these circumstances, biochemical riboflavin deficiency might be relevant. Erythrocyte glutathione reductase (EGR) activity has been examined in a small number of studies in patients with rheumatoid arthritis but with conflicting results. Braven et al reported normal EGR activity in a group of 43 patients with rheumatoid arthritis compared to 29 healthy controls ${ }^{6}$ but Vanella et al described reduced EGR activity in 15 patients with rheumatoid arthritis and Tarp et al recorded a similar finding in nine patients with rheumatoid arthritis. ${ }^{78}$ However, none of these studies has taken account of riboflavin status, which might have influenced their findings.

In the present study, both EGR activity and riboflavin status were comprehensively evaluated in a large group of patients with rheumatoid arthritis and in healthy controls using a functional assay, the EGR activation coefficient (EGRAC), ${ }^{3}$ where EGR is measured in vitro both before and after the addition of exogenous FAD.

\section{Methods \\ SUBJECTS}

Patients had classic or definite rheumatoid arthritis as defined by the American College of Rheumatology criteria. ${ }^{9}$ They were classified as active if they had clinical and laboratory features of active articular inflammation which required initiation or change of disease modifying therapy, or as inactive if they had little clinical or laboratory evidence of articular inflammation. Healthy control subjects were recruited from a local factory workforce undergoing an occupational health examination and were excluded if ongoing ill health was identified. All who reported vitamin 
Measures of disease activity, basal and stimulated erythrocyte glutathione reductase (EGR) activity, and EGR activity coefficient in patients with rheumatoid arthritis and healthy controls. Values are mean ( $95 \%$ confidence intervals)

\begin{tabular}{|c|c|c|c|}
\hline & Active $R A$ & Inactive $R A$ & Controls \\
\hline Pain VAS & $5.8(5.1$ to 6.6$)$ & $2.1(1.3$ to 3.0$)$ & \\
\hline Morning stiffness (hours) & $2.8(1.6$ to 4.0$)$ & $0.4(0.2$ to 0.6$)$ & \\
\hline Grip strength (mm Hg) & 77 (59 to 95$)$ & 135 (107 to 163$)$ & \\
\hline Articular index & $20(15$ to 26$)$ & $6(4$ to 8$)$ & \\
\hline Haemoglobin ( glitre $\left.^{-1}\right)$ & $118(113$ to 122$)$ & 133 (126 to 139$)$ & $144(142$ to 145$)$ \\
\hline $\operatorname{MCV}(\mathrm{fl})$ & $81(79$ to 83$)$ & $87(84$ to 90$)$ & $90(89$ to 91$)$ \\
\hline CRP (mg litre ${ }^{-1}$ ) & $62(46$ to 78$)$ & $7(2$ to 11$)$ & \\
\hline $\operatorname{ESR}(\mathrm{mm} / \mathrm{h})$ & $61(53$ to 69$)$ & 24 (16 to 32$)$ & \\
\hline Basal EGR activity (IU litre ${ }^{-1}$ ) & $7.8(7.3$ to 8.3$)$ & $7.8(7.2$ to 8.3$)$ & 6.7 (6.5 to 6.8$)$ \\
\hline Stimulated EGR activity (IU litre ${ }^{-1}$ ) & $9.6(9.0$ to 10.1$)$ & $8.9(8.3$ to 9.6$)$ & $8.5(8.3$ to 8.7$)$ \\
\hline EGRAC & $1.25(1.21$ to 1.29$)$ & $1.16(1.13$ to 1.20$)$ & $1.29(1.27$ to 1.32$)$ \\
\hline
\end{tabular}

VAS, visual analogue scale; MCV, mean corpuscular volume; CRP, C reactive protein; ESR, erythrocyte sedimentation rate; EGR, erythrocyte glutathione reductase; EGRAC, EGR activation coefficient.

supplementation or excessive alcohol intake were excluded. Demographic details were recorded in all. Patients completed a $10 \mathrm{~cm}$ visual analogue scale for pain; duration of morning stiffness (to nearest half hour), grip strength (mm $\mathrm{Hg})$, and Ritchie articular index ${ }^{10}$ were also measured. All gave informed consent and the study was approved by the hospital ethics committee.

\section{ASSESSMENTS}

Laboratory methods

Haemoglobin and mean corpuscular volume were measured in all using a Coulter counter, model D1 (Coulter Electronics, Luton, England). In addition, serum $\mathrm{C}$ reactive protein, erythrocyte sedimentation rate (ESR), and rheumatoid factor (RF) were measured in patients.

Basal glutathione reductase activity assay-All reagents were obtained from British Drug Houses (Poole, England) unless otherwise stated. Basal EGR activity was measured in patients and controls, as previously described. ${ }^{311}$ In brief, red cells were washed three times in cold $0.15 \mathrm{M}$ saline and $0.5 \mathrm{ml}$ of washed red cells were haemolysed in $9.5 \mathrm{ml}$ distilled water to make a 1:20 haemolysate. Basal activity in $0.1 \mathrm{ml}$ of haemolysate was measured in duplicate in $0.1 \mathrm{M}$ potassium phosphate buffer ( $\mathrm{pH} 7.4$ ) containing $2.3 \mathrm{mM}$ ethylenediaminetetracetic acid (dipotassium salt) and $0.89 \mathrm{mM}$ oxidised GSSG (Sigma, Poole, England) in a reaction initiated by 80 $\mu \mathrm{M}$ reduced nicotinamide adenine dinucleotide phosphate (NADPH) (Sigma) in a total volume of $3.6 \mathrm{ml}$. The cuvettes were incubated for $5 \mathrm{~min}$ at $37^{\circ} \mathrm{C}$ before the addition of NADPH, and the decrease in absorbance over $3 \mathrm{~min}$ was recorded continuously at $340 \mathrm{~nm}$ and $37^{\circ} \mathrm{C}$ using a $\mathrm{KONE}$ discreet analyser. The reduction in absorbance reflects the decrease in NADPH which, in turn, reflects EGR activity and FAD availability, in the following reaction:

$$
\begin{gathered}
\mathrm{EGR} \\
\mathrm{FAD} \\
\mathrm{FAD}
\end{gathered}
$$

Results are expressed as IU litre ${ }^{-1}$ and are corrected for haemoglobin concentration in the haemolysate.

Measurement of riboflavin status-In this assay, EGR activity is measured following the addition of $8 \mu M$ FAD (Sigma) before the addition of NADPH. Stimulated EGR activity represents maximum EGR activity in that subject in the presence of adequate FAD. EGRAC represents the ratio of stimulated to basal EGR activity; a ratio $\geqslant 1.3$ is believed to represent biochemical riboflavin deficiency.

\section{Quality control}

The EGRAC was measured repeatedly, in parallel with patient and control samples, in each of three different samples representing a low, medium, and a high value. The coefficient of variance measured $2.4 \%$ for the low sample (mean EGRAC $=1.22$ ), $3.3 \%$ for the medium sample $(E G R A C=1.45)$, and $3.6 \%$ for the high sample $($ EGRAC $=1.63)$.

\section{STATISTICAL METHODS}

Data were compared using StatviewSE + Graphics statistical software (Abacus Concepts, Berkeley, CA, USA). EGR activity in the general population has a skew distribution ${ }^{12}$; thus data relating to EGR activity were examined using non-parametric statistical methods: the Mann Whitney U test to compare unpaired data and the Kendall Rank correlation test for correlations. Sex distribution and frequency of riboflavin deficiency in different groups were examined using the $\chi^{2}$ test. $P$ values less than 0.05 were considered significant.

\section{Results}

SUBJECTS

Ninety one patients with rheumatoid arthritis, including 57 with active disease, and 220 healthy controls were studied. There were a significantly greater proportion of females in the rheumatoid arthritis groups $(67 \%)$ than among controls $(43 \%)\left(\chi^{2}=14.7, \mathrm{P}=0.0001\right)$. Mean (SD) age was 55 (12) for patients and 44 (13) years for controls. Thus patients were significantly older that controls $(P=0.0001)$. There were no significant differences between the active and inactive rheumatoid arthritis groups by age or sex distribution, but patients with active disease had significantly higher pain score, duration of morning stiffness, articular index, mean corpuscular volume, $\mathrm{C}$ reactive protein, and ESR, and significantly lower grip strength and haemoglobin (all $\mathrm{P}<0.0005$ ), than patients with inactive disease (table). Patients had lower haemoglobin and mean corpuscular volume than controls (both $\mathrm{P}=$ 0.0001). 
BASAL GLUTATHIONE REDUCTASE ACTIVITY

Basal EGR activity results are given in the table. There was no significant difference between basal activity in male or female controls $(P=0.71)$ and only a weak correlation $(\mathrm{T}=0.11, \mathrm{P}=0.02)$ was identified between basal activity and age. Basal EGR activity was significantly higher in both active and inactive rheumatoid arthritis patients than in controls $(P=0.0001)$. There was no significant difference between basal activity in active and inactive rheumatoid arthritis patients.

\section{RIBOFLAVIN STATUS}

Stimulated EGR activity and EGRAC results are given in the table. Stimulated EGR activity was significantly higher in both active and inactive rheumatoid arthritis patients than in controls $(P=0.0001)$. There was no significant difference between stimulated activity in active and inactive rheumatoid arthritis patients. Biochemical riboflavin deficiency (EGRAC $\geqslant 1.3$ ) was identified in 90 controls (41\%) and was significantly more common among women in this group $\left(\chi^{2}=14.3, P=0.0001\right)$. Riboflavin deficiency was identified in 22 patients with rheumatoid arthritis (24\%)-19 with active disease $(33 \%)$ and three with inactive disease $(9 \%)$. The frequency of riboflavin deficiency in active rheumatoid arthritis was similar to that in controls $\left(\chi^{2}=0.79\right)$, but it was less frequent in inactive rheumatoid arthritis than in patients with active disease $\left(\chi^{2}=5.7, P=0.02\right)$ or healthy controls $\left(\chi^{2}=11.7, \mathrm{P}=0.0006\right)$. Riboflavin deficiency was not increased in females with rheumatoid arthritis compared to males.

There was evidence that riboflavin deficiency was associated with greater disease activity in rheumatoid arthritis patients: median pain score, articular index, $\mathrm{C}$ reactive protein, and ESR were significantly higher $\left(7.0,22,47 \mathrm{mg}\right.$ litre $^{-1}$, and $64 \mathrm{~mm} / \mathrm{h}$ respectively) in patients with riboflavin deficiency than in patients without riboflavin deficiency $\left(4.0,11,18 \mathrm{mg}\right.$ litre $^{-1}, 38 \mathrm{~mm} / \mathrm{h}$, respectively) (all $P<0.02$ ). In addition, $C$ reactive protein and ESR correlated weakly ( $T$ $=0.27, \mathrm{P}=0.0002$ and $\mathrm{T}=0.24, \mathrm{P}=0.001$, respectively) with EGRAC.

\section{Discussion}

In our study we measured erythrocyte glutathione reductase activity and riboflavin status in a large group of patients with rheumatoid arthritis and in healthy controls. There were two main findings. Firstly, both basal and stimulated EGR activity are increased in patients compared to controls, as might be expected in response to chronic oxidative stress. Secondly, riboflavin deficiency is no more frequent among patients with rheumatoid arthritis than in our controls, but it is associated with greater disease activity, suggesting that it may impair the response to oxidative stress and facilitate continuing inflammation.

There were significant differences between the patient and control populations. However, age and sex have a negligible effect on EGR activity, as we and others have shown. ${ }^{12}$ Thus the different age and sex distribution of patients and controls is unlikely to explain the differences identified in basal EGR activity and EGRAC. In addition, EGR activity is corrected for haemoglobin concentration in . the haemolysate, thus removing the possible effect of differences between haemoglobin and mean corpuscular volume in patients and controls.

The small number of studies which have measured EGR activity in patients with rheumatoid arthritis have yielded conflicting results. ${ }^{6-8}$ However, no previous study has assessed riboflavin status in rheumatoid arthritis, and there are other important differences between patient and control groups and laboratory methods which may underlie the conflicting findings. In contrast to our present study, Braven et al found no significant difference in basal EGR activity in 43 patients with rheumatoid arthritis compared to 29 healthy individuals. ${ }^{6}$ However, the mean age of their control group was considerably less than their patient group (29 $v 55$ years) and no further details about these individuals were provided. In addition, the coefficient of variance for their EGR assay is relatively high $(12 \%)$. Although no correlation was identified between ESR and EGR in their patients, no further information was available concerning the proportion of patients with active disease in the population studied. In contrast both to the findings in the present study and to that of Braven et al, two small studies have reported basal EGR to be reduced in patients with rheumatoid arthritis. Vanella et al measured EGR in 15 patients with rheumatoid arthritis and 15 age matched controls. ${ }^{7}$ Patients receiving disease modifying drugs were excluded and non-steroidal agents were discontinued for one week before the experimental procedure. However, information concerning disease activity measures was not available. Tarp et al examined nine patients with severe rheumatoid arthritis, defined by extent of joint involvement and laboratory measures of disease activity, and eight healthy controls with a similar age and sex distribution. ${ }^{8}$ Their observation of reduced EGR activity in the small number of patients with active rheumatoid arthritis examined is consistent with the hypothesis that riboflavin deficiency could facilitate continuing inflammation in situations of chronic oxidative stress.

Riboflavin (vitamin B-2) is a water soluble B vitamin that serves as a precursor of $F A D$ and flavin mononucleotide. These two compounds are important coenzymes in a variety of electron transfer reactions during energy producing, biosynthetic, detoxifying, and electron scavenging processes. ${ }^{2}$ Clinical manifestations of riboflavin deficiency are uncommon, but include cheilosis, angular stomatitis, seborrhoeic dermatitis, and other skin changes. The recommended daily dietary allowance ranges from $0.4 \mathrm{mg}$ in infants to $1.8 \mathrm{mg}$ in young male adults and is further increased by pregnancy and lactation. ${ }^{13}$ There are various laboratory methods available to measure riboflavin status, but is generally believed that the functional EGRAC assay has the fewest drawbacks. ${ }^{1314}$ 
The frequency of biochemical riboflavin deficiency varies greatly in different populations studied. While Glatzle et al described biochemical riboflavin deficiency in $32 \%$ of 124 geriatric patients, the frequency of biochemical riboflavin deficiency is reported to be less than $1 \%$ in adults in the United Kingdom, a figure clearly at odds with the frequency among controls in the present study. ${ }^{15}$ These findings suggest under-representation of some sections of the population in cited national data. However, data from our control population, drawn from a local workplace, may be more relevant to our patients with rheumatoid arthritis.

The normal response to chronic oxidative stress, such as sustained physical training, involves increased GSH and EGR activity. ${ }^{4} \mathrm{~A}$ similar pattern of increased EGR activity in patients with rheumatoid arthritis is identified in the present study. However, the relation of disease activity to GSH described by others, and to riboflavin status in the present study, suggests that EGR activity- while increased in patients with rheumatoid arthritis-may remain suboptimal in those with riboflavin deficiency. In these patients, EGR activity may remain inadequate to deal completely with the oxidative stresses involved, facilitating continuing inflammation. There is growing awareness that reactive oxygen species and free radicals may play an important role in mediating cellular injury and tissue damage in rheumatoid arthritis. ${ }^{1}$ In addition, it is acknowledged that riboflavin derivatives are important elements of the protective mechanisms which combat oxidative stress. ${ }^{21316}$ In this context, the findings in this study provide a rationale for evaluating the effect of riboflavin supplementation in patients with active rheumatoid arthritis.
1 Stefanovic-Racic M, Stadler J, Evans CH. Nitric oxide and arthritis. Arthritis Rheum 1993;36:1036-44.

2 Voet D, Voet JG. Biochemistry, 2nd ed. New York: John Wiley \& Co, 1995.

3 Glatzle D, Körner WF, Christeller S, Wiss O. Method for the detection of a biochemical riboflavin deficiency. Stimulation of $\mathrm{NADPH}_{2}$-dependent glutathione reductase from human erythrocytes by FAD in vitro. Investigations on the vitamin $B_{2}$ status in healthy people and geriatric patients. Int $\mathcal{Y}$ Vitam Res 1970;40:166-83.

4 Evelo CTA, Palmen NGM, Artur Y, Janssen GME. Changes in blood glutathione concentrations, and in erythrocyte glutathione reductase and glutathione ticipation in contests. Eur $\mathcal{f}$ Appl Physiol 1992;64:354-8.

5 Munthe E, Kåss E, Jellum E. D-penicillamine-induced increase in intracellular glutathione correlating to clinical response in rheumatoid arthritis. $\mathcal{f}$ Rheumatol 1981; 8(suppl 7):14-19.

6 Braven J, Ansari N, Figgitt DP, Fisher A, Luders C, Hickling $\mathrm{P}$, et al. A comparison of glutathione reductase and $\mathrm{P}$, et al. A comparison of glutathione reductase and glutathione peroxidase activities in patients with rheuma28:212-5.

7 Vanella A, Raqusa N, Campisi A, Sorrenti V, Murabito L, Attaguile $\mathrm{G}$, et al. Antioxidant enzymatic systems in erythrocytes from patients with rheumatoid arthritis. $\mathrm{Med} S \mathrm{Sc}$ Res 1987;15:1187-8.

8 Tarp U, Stengaard-Pedersen K, Hansen JC, Thorling EB Glutathione redox cycle enzymes and selenium in severe Glutathione redox cycle enzymes and selenium in severe
rheumatoid arthritis: lack of antioxidative response to selenheumatoid arthritis: lack of antioxidative response to selenium supplementation in polym
Ann Rheum Dis 1992;51:1044-9.

9 Ropes MW, Bennett GA, Cobb S, Jacox R, Jessar rheumatoid arthritis. 1958 revision of diagnostic criteria for rheumatoid arthritis. Bull Rheum Dis 1958;9:175-6.

10 Ritchie DM, Boyle JA, McInnes JM, Jasani MK, Dalakas TG, Grieveson P, et al. Clinical studies with an articular index for the assessment of joint tenderness in patient with rheumatoid arthritis. $Q \mathcal{F} M e d$ 1968;147:393-406.

11 Adelekan DA, Thurnham DI. Effects of combined riboflavin and iron deficiency on the hematological status and tissue iron concentrations of the rat. $f$ Nutr 1986; 116:1257-65.

12 Salkie ML, Simpson E. The erythrocyte glutathione reductase activity (E.C. 1.6.4.2) of healthy blood donors. $\mathcal{f}$ Clin Pathol 1970;23:708-10.

13 Food and Nutrition Board Subcommitee. Recommended dietary allowances, 10th ed. Washington DC: National Academy Press 1989, 132-6.

14 Campbell TC, Brun T, Junshi C, Zulin F, Parpia B. Questioning riboflavin recommendations on the basis of survey in China. Am $\mathfrak{f}$ Clin Nutr 1990:51:436-45.

15 Gregory JR, Foster K, Tyler H, Wiseman M. The dietary and nutritional survey of British adults. London: HMSO, 1990

16 Christensen HN. Riboflavin can protect tissues from oxidative injury. Nutr Rev 1993;51:149-50. 